\title{
Diagnostic Performance of Ultrafast Brain MRI for Evaluation of Abusive Head Trauma
}

\author{
(D) S.F. Kralik, (D) M. Yasrebi, (D) N. Supakul, (D) C. Lin, (D) L.G. Netter, (D).A. Hicks, DR.A. Hibbard, (D) L.L. Ackerman, (D) M.L. Harris, and \\ (D).Y. Ho
}

\begin{abstract}
BACKGROUND AND PURPOSE: MR imaging with sedation is commonly used to detect intracranial traumatic pathology in the pediatric population. Our purpose was to compare nonsedated ultrafast MR imaging, noncontrast head CT, and standard MR imaging for the detection of intracranial trauma in patients with potential abusive head trauma.
\end{abstract}

MATERIALS AND METHODS: A prospective study was performed in 24 pediatric patients who were evaluated for potential abusive head trauma. All patients received noncontrast head CT, ultrafast brain MR imaging without sedation, and standard MR imaging with general anesthesia or an immobilizer, sequentially. Two pediatric neuroradiologists independently reviewed each technique blinded to other modalities for intracranial trauma. We performed interreader agreement and consensus interpretation for standard MR imaging as the criterion standard. Diagnostic accuracy was calculated for ultrafast MR imaging, noncontrast head CT, and combined ultrafast MR imaging and noncontrast head CT.

RESULTS: Interreader agreement was moderate for ultrafast MR imaging ( $\kappa=0.42$ ), substantial for noncontrast head CT $(\kappa=0.63)$, and nearly perfect for standard MR imaging $(\kappa=0.86)$. Forty-two percent of patients had discrepancies between ultrafast MR imaging and standard MR imaging, which included detection of subarachnoid hemorrhage and subdural hemorrhage. Sensitivity, specificity, and positive and negative predictive values were obtained for any traumatic pathology for each examination: ultrafast MR imaging ( $50 \%, 100 \%, 100 \%, 31 \%)$, noncontrast head CT (25\%, 100\%, 100\%, $21 \%$ ), and a combination of ultrafast MR imaging and noncontrast head CT (60\%, 100\%, 100\%, 33\%). Ultrafast MR imaging was more sensitive than noncontrast head CT for the detection of intraparenchymal hemorrhage $(P=.03)$, and the combination of ultrafast MR imaging and noncontrast head CT was more sensitive than noncontrast head CT alone for intracranial trauma $(P=.02)$.

CONCLUSIONS: In abusive head trauma, ultrafast MR imaging, even combined with noncontrast head CT, demonstrated low sensitivity compared with standard MR imaging for intracranial traumatic pathology, which may limit its utility in this patient population.

ABBREVIATIONS: $\mathrm{AHT}=$ abusive head trauma; $\mathrm{nHCT}=$ noncontrast head $\mathrm{CT}$; stMRI = standard MR imaging; ufMRI = ultrafast $\mathrm{MR}$ imaging

$\mathbf{T}$ he incidence of abusive head trauma (AHT) in the United States from 2000 to 2009 was 39.8 per 100,000 children younger than 1 year of age and 6.8 per 100,000 children 1 year of age. ${ }^{1}$ The outcomes of patients with AHT are worse than those of children with accidental traumatic brain injury, including higher

Received September 15, 2016; accepted after revision December 5.

From the Department of Radiology and Imaging Sciences (S.F.K., M.Y., N.S., C.L., L.G.N., C.Y.H.), Child Protective Program (R.A. Hicks, R.A. Hibbard), Departments of Neurological Surgery (L.L.A.) and Neurology (M.L.H.), Indiana University School of Medicine, Indianapolis, Indiana.

Paper previously presented in part at: Annual Meeting of the American Society of Neuroradiology and the Foundation of the ASNR Symposium, April 25-30, 2015; Chicago, Illinois.

Please address correspondence to Chang Ho, MD, MRI Department, 705 Riley Hospital Dr, Indiana University School of Medicine, Indianapolis, IN 46202; e-mail: cyho@iupui.edu

http://dx.doi.org/10.3174/ajnr.A5093 rates of mortality and permanent disability from neurologic impairment. ${ }^{2-5}$ The diagnosis of AHT is frequently not recognized when affected patients initially present to a physician, and up to $28 \%$ of children with a missed AHT diagnosis may be re-injured, leading to permanent neurologic damage or even death. ${ }^{6}$ Because neuroimaging plays a central role in AHT, continued improvement in neuroimaging is necessary.

Common neuroimaging findings of AHT include intracranial hemorrhage, ischemia, axonal injury, and skull fracture, with advantages and disadvantages for both $\mathrm{CT}$ and MR imaging for the detection of AHT. ${ }^{7}$ A noncontrast head CT (nHCT) is usually the initial imaging study in suspected AHT due to its high sensitivity for the detection of acute hemorrhage and fracture and the high level of accessibility from the emergency department, and it can be performed quickly and safely without the need for special monitoring equipment. ${ }^{8,9}$ The disadvantages of CT include ionizing 
Table 1: Ultrafast MRI brain protocols

\begin{tabular}{lcccccc}
\hline & \multicolumn{9}{c}{ Parameters } & \\
\cline { 2 - 6 } \multicolumn{1}{c}{ Sequence } & $\begin{array}{c}\text { Magnet } \\
\text { Strength }\end{array}$ & TE (ms) & TR (ms) & Matrix & $\begin{array}{c}\text { Section } \\
\text { Thickness } \\
(\mathbf{m m})\end{array}$ & $\begin{array}{c}\text { Total Time: } \\
\text { 1.5T: 1m 43s; } \\
\text { 3T: 1m 54s }\end{array}$ \\
\hline Axial T2 HASTE & $1.5 T$ & 96 & 550 & $192 \times 154$ & 4 & $23 \mathrm{~s}$ \\
& $3 \mathrm{~T}$ & 98 & 536 & $192 \times 154$ & 4 & $19 \mathrm{~s}$ \\
Coronal T2 HASTE & & 96 & 550 & $123 \times 192$ & 4 & $23 \mathrm{~s}$ \\
& & 98 & 536 & $123 \times 192$ & 4 & $19 \mathrm{~s}$ \\
Axial DWI & & 77 & 4508 & $128 \times 128$ & 4 & $36 \mathrm{~s}$ \\
& & 78 & 12,600 & $128 \times 128$ & 4 & $46 \mathrm{~s}$ \\
Axial EPI T2* & & 39 & 4190 & $192 \times 154$ & 4 & $21 \mathrm{~s}$ \\
& & 39 & 3350 & $192 \times 154$ & 4 & $30 \mathrm{~s}$ \\
\hline
\end{tabular}

Note:- $m$ indicates minute; $s$, second.

radiation, particularly in children, and the reduced sensitivity in detecting microhemorrhages, axonal injury, and acute ischemia compared with MR imaging. ${ }^{10}$

MR imaging is frequently performed in AHT and adds additional information in $25 \%$ of all children with abnormal findings on the initial CT scan. ${ }^{11}$ Brain MR imaging can also be useful for identifying bridging vein thrombosis, differentiating subdural fluid collections from enlarged subarachnoid spaces, characterizing the signal of subdural blood, and demonstrating membrane formation within subdural collections. ${ }^{12-16}$ Brain MR imaging findings have correlated with poor outcomes associated with findings on diffusion-weighted imaging and susceptibilityweighted imaging in AHT; however, disadvantages of MR imaging continue to include the need for sedation in children and compatible monitoring equipment. ${ }^{17-22}$ Although there is greater accessibility of CT compared with MR imaging, the availability of MR imaging is relatively high and imaging techniques that allow neuroimaging in patients with potential AHT without sedation would be valuable, particularly given the potential adverse effects of sedation on the developing brain. ${ }^{23,24}$

A potential solution for diagnostic-quality brain MR imaging without sedation in AHT is the use of ultrafast MR imaging (ufMRI) sequences, also termed "fast MR imaging," "quick MR imaging," or "rapid MR imaging." Ultrafast MR imaging uses pulse sequences that rapidly acquire images, potentially reducing motion artifacts and the need for sedation. ufMRI has been most commonly used in pediatric neuroradiology for the evaluation of intracranial shunts in children with hydrocephalus, and most of the reported ufMRI brain protocols include only multiplanar T2weighted HASTE sequences. ${ }^{25-34}$ Consequently, previously reported limitations of ufMRI in detecting intracranial hemorrhage is primarily due to the lack of blood sensitive sequences. ${ }^{35}$

Recently, an ufMRI protocol incorporating sequences in addition to $\mathrm{T} 2$ sequences has been reported in pediatric patients with trauma. ${ }^{36}$ This study did not compare findings with those of a standard MR imaging (stMRI) and included a wider age range of pediatric patients, so the value of ufMRI in pediatric abusive head trauma remains uncertain. ${ }^{36}$ Therefore, the purpose of our study was to evaluate an ufMRI brain protocol performed without sedation for feasibility in terms of scanning time and diagnostic value as well as diagnostic accuracy compared with nHCT and stMRI of the brain for the detection of intracranial traumatic pathology in patients with suspected AHT.

\section{MATERIALS AND METHODS}

Following institutional review board approval, a prospective study was performed from March 2014 through March 2015, evaluating the diagnostic performance of an ufMRI of the brain performed at a tertiary children's hospital in 24 infants who underwent MR imaging for the indication of potential AHT. Infants were eligible for enrollment if they had presented acutely to an emergency department, had undergone an nHCT within the preceding 48 hours either performed at a referring institution or our institution, and were not intubated or sedated for clinical reasons and MR imaging of the head had been requested to further evaluate the patient for potential AHT. The following clinical data were collected for each subject: age, sex, and presentation pediatric Glasgow Coma Scale score. For all patients, an ufMRI brain protocol was performed without sedation and, depending on age, with or without an MRI compatible immobilizer (MedVac Infant Immobilizer; CFI Medical, Fenton, Michigan). At our institution, an immobilizer is routinely used for infants younger than 3 months of age. The ufMRI was immediately followed by an stMRI of the brain with continued use of an immobilizer or with general anesthesia, with a maximum of time interval between the completion of ufMRI and the start of stMRI of 25 minutes in patients requiring sedation. Patients were not excluded if the ufMRI was nondiagnostic but were excluded if stMRI sequences were nondiagnostic.

MR imaging was performed with $1.5 \mathrm{~T}$ or $3 \mathrm{~T}$ scanners (Avanto and Verio; Siemens, Erlangen, Germany). The ufMRI and stMRI protocol details are shown in Tables 1 and 2. MR imaging technologists were instructed to repeat an ufMRI sequence only once if there were severe motion artifacts. Technical parameters for nHCT were the following: 100-120 kV(peak); 145-185 mA; and CT dose index, 17.1-29.4 mGy.

Two board-certified fellowship-trained pediatric neuroradiologists (S.F.K., C.Y.H.) with Certificates of Added Qualification in neuroradiology with 3 and 8 years of experience, respectively, independently reviewed the ufMRIs followed by a review of the stMRIs. Reviewing the ufMRI first without the results of the stMRI allowed a blinded evaluation of the ufMRI. These were reviewed by the same 2 pediatric neuroradiologists at a separate time following a 2-month interval from the MR imaging analysis, to avoid memory bias for nHCT. Axial soft-tissue-algorithm $\mathrm{nHCT}$ at 5-mm section thickness were included for review. Coronal and sagittal reformats were not available in all cases and were not included in the evaluation. The pediatric neuroradiologists were aware that the clinical indication was for evaluation of potential AHT but were otherwise blinded to the final clinical interpretation and additional clinical and radiologic information of the patient, including skeletal survey results.

UfMRIs, nHCTs, and stMRIs were reviewed for subjective diagnostic quality (diagnostic versus nondiagnostic), and specific assessment was recorded for the following: subdural fluid collection (unilateral, bilateral, tentorial, presence of subdural fluid- 
Table 2: stMRI brain protocols

\begin{tabular}{|c|c|c|c|c|c|c|}
\hline \multirow[b]{2}{*}{ Sequence } & \multicolumn{5}{|c|}{ Parameters } & \multirow[b]{2}{*}{$\begin{array}{l}\text { Total Time: } \\
\text { 1.5T: } 17 \mathrm{~m} \mathrm{15s} \text {; } \\
3 \mathrm{~T}: 14 \mathrm{~m} \mathrm{42s}\end{array}$} \\
\hline & $\begin{array}{l}\text { Magnet } \\
\text { Strength }\end{array}$ & TE (ms) & TR (ms) & Matrix & $\begin{array}{c}\text { Section } \\
\text { Thickness } \\
\text { (mm) }\end{array}$ & \\
\hline Sagittal 3D T1 & $1.5 \mathrm{~T}$ & 2.98 & 2180 & $192 \times 256$ & 1.2 & $3 \mathrm{~m} \mathrm{53s}$ \\
\hline MPRAGE & $3 \mathrm{~T}$ & 2.18 & 1460 & $251 \times 256$ & 0.9 & $3 \mathrm{~m} 16 \mathrm{~s}$ \\
\hline \multirow[t]{2}{*}{ Axial T2 TSE } & & 99 & 3950 & $320 \times 320$ & 2 & $\operatorname{lm} 51 \mathrm{~s}$ \\
\hline & & 116 & 3980 & $307 \times 384$ & 2 & $2 \mathrm{~m} \mathrm{12s}$ \\
\hline \multirow[t]{2}{*}{ Coronal T2 TSE } & & 109 & 3870 & $320 \times 320$ & 2 & $2 \mathrm{~m} \mathrm{12s}$ \\
\hline & & 116 & 3520 & $320 \times 320$ & 2 & $4 \mathrm{~m} 6 \mathrm{~s}$ \\
\hline \multirow[t]{2}{*}{ Axial T2 FLAIR } & & 152 & 10,000 & $256 \times 256$ & 4 & $3 \mathrm{~m} \mathrm{Os}$ \\
\hline & & 107 & 7000 & $180 \times 320$ & 4 & $\operatorname{lm} 24 s$ \\
\hline \multirow[t]{2}{*}{ Axial DWI } & & 77 & 4508 & $128 \times 128$ & 4 & $36 s$ \\
\hline & & 78 & 12,600 & $128 \times 128$ & 4 & $46 s$ \\
\hline \multirow[t]{2}{*}{ Axial SWI } & & 40 & 49 & $195 \times 320$ & 1.5 & $5 m 43 s$ \\
\hline & & 40 & 27 & $182 \times 256$ & 1.5 & $2 \mathrm{~m} \mathrm{58s}$ \\
\hline
\end{tabular}

Note:- $m$ indicates minute; s, second.

fluid levels, presence of subdural membrane formation/subdural septation), subarachnoid hemorrhage, epidural hemorrhage, intraventricular hemorrhage, intraparenchymal hemorrhage, cytotoxic edema, nonhemorrhagic vasogenic parenchymal edema, parenchymal lacerations, hydrocephalus, midline shift, herniation (uncal, subfalcine, tonsillar), enlarged subarachnoid spaces, and encephalomalacia.

Subdural fluid collections were defined as fluid collections located under the dura along the convexities, falx, or tentorium. Fluid-fluid levels were defined as a difference in signal intensity or density that had a meniscus/layering pattern. Subdural membrane formation was defined as an identifiable line/band that separated a subdural fluid collection into $>1$ compartment. Subarachnoid hemorrhage was identified as blood localized within the subarachnoid space including the basal cisterns or that was identified as hyperattenuation on CT and hyperintense signal on FLAIR imaging or hypointense signal on $\mathrm{T} 2 * /$ susceptibilityweighted imaging. Intraparenchymal hemorrhage was defined as intraparenchymal hyperattenuation on CT and focal intra-axial signal abnormality with either low signal on T2-weighted, T2*, or susceptibility-weighted images or high signal intensity on T1weighted images. Cytotoxic edema was defined as an area demonstrating low attenuation on CT involving gray matter and high signal intensity on DWI with low signal intensity on the corresponding apparent diffusion coefficient map and included diffuse axonal injury and vascular infarct. Nonhemorrhagic vasogenic parenchymal edema was defined as low attenuation on CT sparing the gray matter and abnormal T2 signal hyperintensity without associated intraparenchymal hemorrhage or cytotoxic edema as defined above. Parenchymal lacerations were defined as a parenchymal cleft containing CSF and/or hemorrhage that did not correspond to a normal anatomic structure such as a sulcus. Enlarged subarachnoid spaces were defined as subarachnoid spaces measuring $>4 \mathrm{~mm}$ in thickness. Encephalomalacia was defined as a focal loss of brain volume involving the cortex identified on any sequence.

On completion of review of the nHCTs, ufMRIs and stMRIs, discrepancies between neuroradiologists were resolved by discussion to establish a consensus interpretation. For the calculation of concordance, an examination was considered "concordant" if all findings were in agreement and "discordant" if there was any disagreement for any of the pathologic categories. $\kappa$ values $<0$ were considered no agreement; $0-0.20$, as slight agreement; 0.21-0.40, as fair agreement; $0.41-0.60$, as moderate agreement; $0.61-0.80$, as substantial agreement; and $0.81-1$, as almost perfect agreement. ${ }^{37}$ Sensitivity, specificity, and positive and negative predictive values for consensus interpretation for ufMRI, nHCT, and ufMRI combined with nHCT, respectively, were calculated compared with consensus stMRI as the criterion standard. The McNemar test was used to assess significance of the discordance rate compared with the criterion standard for each pathologic entity and the changes in sensitivity among ufMRI, nHCT, and combined ufMRI with nHCT. Statistics were performed by using MedCalc statistical software, Version 14.12.0 (MedCalc Software, Mariakerke, Belgium), with $P<.05$ considered statistically significant.

\section{RESULTS}

The median subject age was 4 months (range, 9 days to 31 months), and the male/female ratio was 2:1. The median presentation pediatric Glasgow Coma Scale score was 15 (range, 13-15). As per study protocol, no sedation was performed during ufMRIs of the brain for all 24 patients. stMRI was performed with an immobilizer in 15/24 (63\%) patients and with general anesthesia for $9 / 24(37 \%)$ patients. ufMRI was performed without sedation in all 24 patients, required less than 2 minutes to acquire all of the imaging sequences, and was of diagnostic quality in all patients, while stMRI required general anesthesia in 9 of 24 patients to achieve diagnostic quality and required approximately $15 \mathrm{~min}$ utes to acquire all of the imaging sequences. ufMRI sequences and stMRI sequences were considered diagnostic in all patients by both neuroradiologists. Four individual ultrafast MRI sequences were repeated in 3/24 scans compared with a repeat of 11 stMRI sequences in 6/24 scans. All nHCTs were of acceptable diagnostic quality.

A summary of the prevalence of imaging findings identified on stMRI is listed in Table 3. The overall prevalence of patients with an abnormal intracranial trauma finding on stMRI was $83.3 \%$. Binary interreader agreement for complete agreement versus any discrepant finding was moderate for ufMRI $(\kappa=0.42 ; 95 \% \mathrm{CI}$, $0-0.87)$, substantial for nHCT $(\kappa=0.63 ; 95 \% \mathrm{CI}, 0.30-0.96)$, and nearly perfect for stMRI $(\kappa=0.86 ; 95 \% \mathrm{CI}, 0.60-1)$. Only 1 patient had an interreader discrepancy on stMRI, which involved the presence of old blood products along the tentorium.

Discrepancy rates for individual findings on the consensus interpretation for ufMRI and nHCT compared with stMRI are listed in Table 4. The only significant discrepancy rate by pathology was the detection of intraparenchymal hemorrhage on nHCT compared with stMRI $(P=.03)$. For the total discrepancy rates per examination type, there was significance for consensus ultrafast MRI $(P=.004)$, nHCT $(P=.0003)$, and combined ufMRI and $\operatorname{nHCT}(P=.01)$ compared with the criterion standard stMRI. 
Discrepancies that consensus ufMRI missed but consensus stMRI detected included the following: 4 patients with subarachnoid hemorrhage, 3 patients with bilateral subdural fluid collections in which 1 collection was not identified, 2 patients with a fluid-fluid level in a subdural collection, and 3 patients with ten-

Table 3: Prevalence of imaging findings per patient on stMRI

\begin{tabular}{lc}
\hline \multicolumn{1}{c}{ Finding } & Prevalence \\
\hline Subdural collection & $11 / 24(46 \%)$ \\
Bilateral subdural collection & $10 / 11(44 \%)$ \\
Subarachnoid hemorrhage & $8 / 24(33 \%)$ \\
Intraparenchymal hemorrhage & $7 / 24(29 \%)$ \\
Intraventricular hemorrhage & $1 / 24(4 \%)$ \\
Epidural hemorrhage & $3 / 24(13 \%)$ \\
Cytotoxic edema & $4 / 24(17 \%)$ \\
Parenchymal laceration & $0 / 24(0 \%)$ \\
Vasogenic edema & $2 / 24(8 \%)$ \\
Herniation or midline shift & $0 / 24(0 \%)$ \\
Hydrocephalus & $0 / 24(0 \%)$ \\
Encephalomalacia & $2 / 24(8 \%)$ \\
Large subarachnoid spaces & $5 / 24(21 \%)$ \\
Total No. of patients with any & $20 / 24(83 \%)$ \\
abnormal finding & \\
\hline
\end{tabular}

torial subdural hemorrhage. ufMRI demonstrated complete agreement between both reviewers; and the stMRI, for the presence of at least 1 subdural collection, intraventricular hemorrhage, parenchymal laceration, enlarged subarachnoid spaces, encephalomalacia, intraparenchymal hemorrhage, herniation or midline shift, and hydrocephalus. There were no abnormal findings described on ultrafast MRI that were normal findings on stMRI. Examples of ufMRI findings compared with stMRI findings are seen in Figs 1-3.

The diagnostic accuracy of consensus comparisons for each test for detecting any intracranial traumatic pathology with the criterion standard stMRI is listed in Table 5. The differences in the resulting sensitivity of ufMRI versus nHCT and ufMRI versus combined ufMRI with nHCT were not statistically significant $(P=.13, P=$ .48); however, the difference in the sensitivity of combined ufMRI with $\mathrm{nHCT}$ versus $\mathrm{nHCT}$ alone was statistically significant $(P=.02)$.

\section{DISCUSSION}

In this study, we demonstrate that an ufMRI can be reproducibly performed in pediatric patients referred for potential AHT, with subjective diagnostic quality and without sedation. The lack of the need for sedation is considered a primary advantage of ufMRI, and this may allow more institutions to perform brain MRIs on these patients without the requirement of anesthesiology. Indeed, at many institutions that have an MR imaging scanner and even those with 24/7 MR imaging technologist availability, anesthesiology can become a limiting factor for MR imaging in pediatric patients. However, ufMRI may be of little benefit if patients are intubated for clinical reasons because stMRI sequences could be performed without loss of spatial resolution.

Although feasible, ufMRI demonstrated decreased interreader concordance between the reviewers compared with stMRI. Several of the discrepancies could be identified in retrospect on the ufMRI but were likely missed due to thicker sectioning. The most frequent discrepant finding involved detection and localization of subarachnoid hemorrhage, which was better appreciated on SWI than ultrafast axial $\mathrm{T} 2{ }^{*}$ images, likely due to differences in both spatial resolution and signal intensity. Although many missed findings on ufMRI can be retrospectively appreciated, given that both reviewers have experience in pediatric neuroimaging, the decreased interreader concordance is a limitation of ufMRI compared with stMRI.

Compared with nHCT, ultrafast MRI demonstrated similar discrepancy
FIG 1. A 4-month-old infant with suspected abusive head trauma found to have bilateral subdural collections identified on coronal T2 TSE $(A)$; however, the right subdural collection was not prospectively identified on ultrafast coronal T2 HASTE (B). 
rates for the detection of subdural and subarachnoid blood, but had significantly improved detection of intraparenchymal hematoma. These findings are likely due to $\mathrm{T} 2{ }^{*}$ sequences, which detect not only acute blood, which would be bright on nHCT, but also chronic hemosiderin, which would be essentially undetectable on nHCT. Although signal loss on $\mathrm{T}^{*}$ cannot differentiate the chronicity of blood, the detection of blood products not seen on $\mathrm{nHCT}$ indicates previous injury and would be helpful when assessing AHT. We did not find differences in the detection of intraparenchymal hemorrhage between ufMRI and stMRI in these patients; however, previous reports have demonstrated a greater sensitivity of SWI compared with gradient recalled-echo for the detection of cerebral microhemorrhage; therefore, we suspect similarly that the ultrafast $\mathrm{T} 2 *$ images will be less sensitive to the detection of cerebral microhemorrhage compared with SWI in a larger cohort. ${ }^{38}$ The lack of significance for the detection of cytotoxic edema and enlarged subarachnoid spaces between ufMRI and nHCT was not expected because DWI is more sensitive to cytotoxic edema than CT, and T2 HASTE images show the bridging veins within the subarachnoid space more clearly. This unexpected finding may be due to the lower prevalence of these entities in our patient cohort.

Our rationale for combining nHCT and ufMRI is the theoretic algorithm of using both examinations as a potential replacement for stMRI, with nHCT providing greater sensitivity for skull fractures and ufMRI, for parenchymal injury. While this combination does improve sensitivity compared with nHCT alone and raises
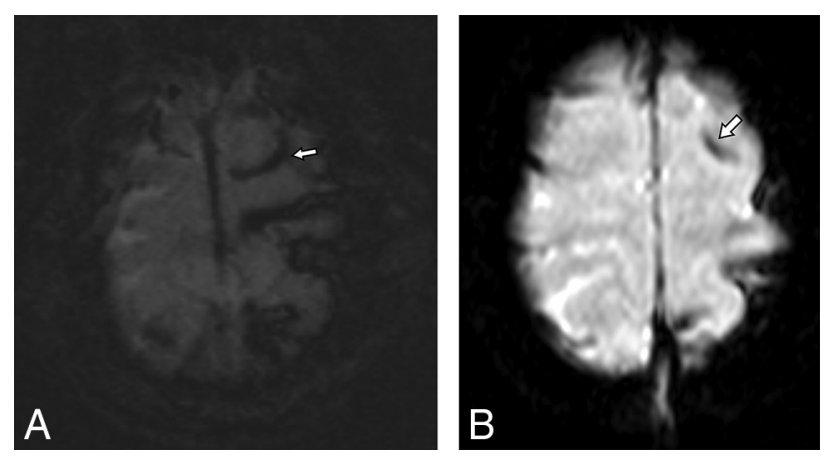

FIG 2. A 31-month-old child with a suspected abusive head trauma with a subdural hematoma (not shown) found to have subarachnoid hemorrhage in the sulci of the left superior frontal and parietal lobes on axial SWI $(A)$, which was prospectively detected by only 1 reviewer on ultrafast axial EPI T2* $(B)$.
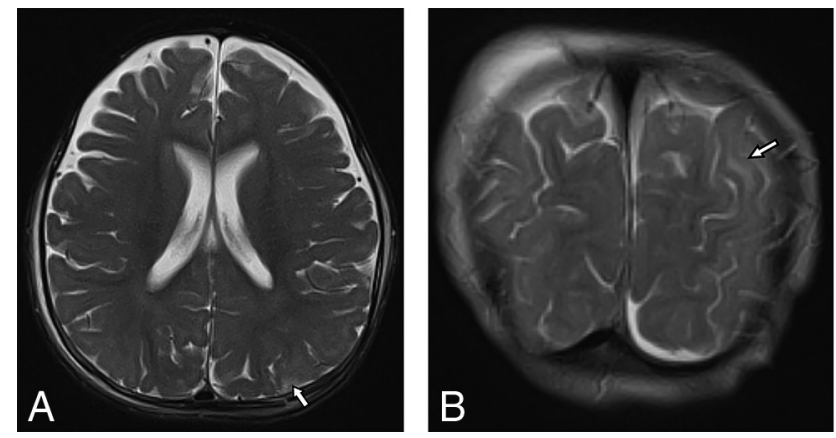

sensitivity slightly for intracranial pathology compared with ultrafast MRI alone, the overall low sensitivity likely reflects the high sensitivity of SWI on stMRI to small hemorrhages overall, particularly in the subarachnoid space. The decreased sensitivity of ufMRI, nHCT, and the combination of the 2 compared with criterion standard stMRI limits our ability to recommend the use of ufMRI in potential AHT. Institutions that incorporate ufMRI for pediatric patients with trauma should be aware of this potential limitation, and we suggest that if an alternative ufMRI protocol is used, a comparison should be made with an stMRI to assess the accuracy of the ufMRI.

Discrepancies with ufMRI findings may be reduced if these studies are performed more frequently, allowing increased familiarity of the radiologist to the subtleties of ufMRI findings, or they could be avoided by reviewing these studies in consensus. Another possibility would be limiting the use of ufMRI for specific indications such as differentiation of enlarged subarachnoid spaces versus chronic subdural hematomas on nHCT or screening for intracranial trauma in patients with low clinical suspicion for AHT, which can be followed by a later conventional MR imaging if necessary. ufMRI was very accurate for the differentiation of enlarged subarachnoid spaces from subdural collections, a common difficulty with nHCT. If ufMRI is incorporated into clinical use, we recommend a period in which side-by-side analysis with stMRIs is performed before completely replacing stMRI sequences and a low threshold for recommending stMRI.

We could have chosen a broader population to study, particularly any child who came into the emergency department for head trauma, accidental or abusive. However, the included patients in our study are an ideal patient population because of the younger age range, with a higher likelihood of requiring sedation for MR imaging. However, the goal of MR imaging in AHT is not necessarily for acute patient management but for a highly sensitive imaging technique to document intracranial injury in a medicolegal context. One could argue that needing a high level of sensitivity requires neuroimaging with the least amount of error in this patient population and is an ideal challenge to the concept of a fast MR imaging not needing sedation. Because of the need for detail with regard to medicolegal issues, we did not theorize whether the misses on ufMRI without an stMRI would lead to immediate poor patient outcome. Because most of the discrepancies were smaller findings, we would expect a limited effect on
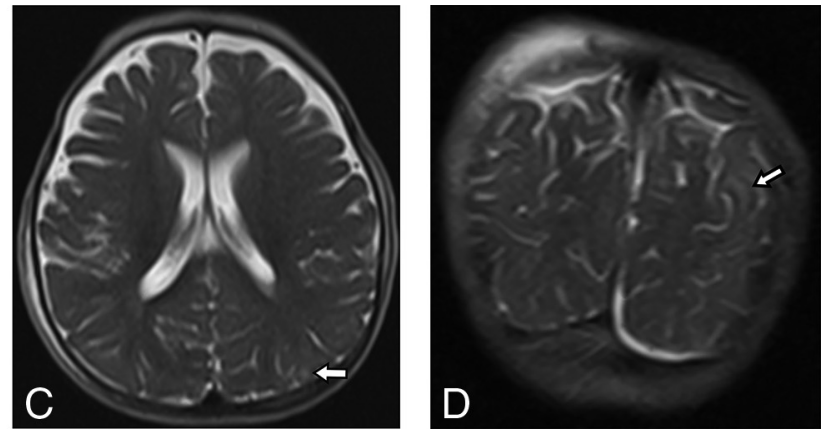

FIG 3. A 10-month-old child with suspected abusive head trauma found to have subtle parenchymal edema identified in the left parietal lobe on axial and coronal T2 TSE ( $A$ and $B$ ), which was not prospectively identified on ultrafast axial or coronal HASTE (C and D). 
Table 5: Diagnostic performance of consensus ufMRI, $\mathrm{nHCT}$, and combined ufMRI and $\mathrm{nHCT}$ compared with stMRI ${ }^{\mathrm{a}}$

\begin{tabular}{lcccc}
\hline & Sensitivity & Specificity & PPV & NPV \\
\hline ufMRI & $50 \%$ & $100 \%$ & $100 \%$ & $31 \%$ \\
& $(27 \%-73 \%)$ & $(40 \%-100 \%)$ & $(69 \%-100 \%)$ & $(8 \%-58 \%)$ \\
nHCT & $25 \%$ & $100 \%$ & $100 \%$ & $21 \%$ \\
& $(9 \%-49 \%)$ & $(40 \%-100 \%)$ & $(48 \%-100 \%)$ & $(6 \%-46 \%)$ \\
Combined ultrafast with nHCT & $60 \%$ & $100 \%$ & $100 \%$ & $33 \%$ \\
& $(36 \%-81 \%)$ & $(40 \%-100 \%)$ & $(74 \%-100 \%)$ & $(10 \%-65 \%)$ \\
\hline
\end{tabular}

Note:-PPV indicates positive predictive value; NPV, negative predictive value.

a Parentheses denote $95 \%$ confidence intervals.

immediate patient outcome, not considering the known poor long-term outcomes of a child at risk for abuse. In this regard, ufMRI could play a larger role in screening for intracranial pathology when AHT is unlikely.

\section{Limitations}

One limitation of this study is the relatively small sample size. A larger number of patients or a multicenter study may help further the understanding of findings on ufMRI that are reproducibly identified or missed compared with stMRI. Also, the nHCT technique was variable due to inclusion of examinations from referring institutions rather than repeating the $\mathrm{nHCT}$ and exposing the patient to additional radiation. Decreasing doses on head CT lessen the signal-to-noise ratio and possibly sensitivity to intracranial pathology. However, our institution is a firm adherent to the Image Gently pledge of the Alliance for Radiation Safety in Pediatric Imaging ${ }^{39}$ and has a consistently lower dose than our referring institutions. Increasing the radiation dose at the cost of potential increased risk of malignancy seems counterproductive in this sensitive patient population. Finally, the study was performed across both 1.5T and 3T scanners, which have signal-tonoise differences. Because the ultrafast MRI and stMRI examinations were performed on the same magnet, this dichotomy in methodology likely has less effect on our results.

A few of our pathologic categories had zero prevalence in this small patient sample, particularly hydrocephalus, herniation and midline shift, and parenchymal lacerations. This is likely due to the exclusion criterion of intubation, resulting in a neurologically intact patient cohort. Hydrocephalus and mass effect causing herniation and midline shift would not be expected to be missed on ufMRI, given the gross morphologic changes to the brain. However, parenchymal lacerations or subcortical tears are uncommon-but-specific injuries for AHT in very young infants due to immature myelination of the subcortical white matter. Given the small size of these lesions, the sensitivity of ufMRI for this finding is uncertain.

Finally, T1- and T2-weighted FLAIR sequences were conspicuously absent in our ultrafast protocol. These would likely increase both concordance and sensitivity for intracranial pathology. However, these sequences are also sensitive to patient motion due to the length of the acquisition, even with decreasing NEX and matrix size. Optimization of time-versus-image signal and resolution by altering these parameters is a further area of study. Furthermore, motioncorrection techniques, such as radial $k$-space acquisition, may also be beneficial despite the longer time for acquisition.

\section{CONCLUSIONS}

Diagnostic-quality ufMRI of the brain can be reliably obtained without sedation in patients with potential AHT, and ufMRI requires a very short amount of time to acquire compared with stMRI. However, ufMRI of the brain, as evaluated in our study, demonstrated greater discrepancy between neuroradiologists and had a low sensitivity for intracranial trauma findings, particularly subarachnoid hemorrhage, even when combined with nHCT. These findings limit the use of ufMRI, or a combination of ufMRI and nHCT, as a replacement examination for an stMRI in the imaging work-up of AHT.

Disclosures: Chen Lin-UNRELATED: Grants/Grants Pending: Siemens.* Ralph A Hicks—UNRELATED: Expert Testimony: University Pediatric Associates Inc, ${ }^{*}$ Comments: Dr Hicks has testified as an expert witness in cases involving suspected child abuse/neglect. Roberta A. Hibbard-UNRELATED: Expert Testimony: Department of Child Services, State of Indiana, Comments: child abuse consultation for attorneys and child protection services*; Grants/Grants Pending: Department of Child Services, Criminal Justice Institute*; Payment for Lectures including Service on Speakers Bureaus: Child First Indiana, Comments: child abuse interviewing; Royalties: Elsevier, Comments: child abuse chapter in dental text; Payment for Development of Educational Presentations: Indiana University School of Medicine, Comments: part of grants. * Money paid to the institution.

\section{REFERENCES}

1. Niederkrotenthaler T, Xu L, Parks SE, et al. Descriptive factors of abusive head trauma in young children: United States, 2000-2009. Child Abuse Negl 2013;37:446-55 CrossRef Medline

2. Duhaime AC, Christian C, Moss E, et al. Long-term outcome in infants with the shaking-impact syndrome. Pediatr Neurosurg 1996; 24:292-98 Medline

3. Chevignard MP, Lind K. Long-term outcome of abusive head trauma. Pediatr Radiol 2014;44(suppl 4):S548-58 CrossRef Medline

4. Reece RM, Sege R. Childhood head injuries: accidental or inflicted? Arch Pediatr Adolesc Med 2000;154:11-15 Medline

5. Sills MR, Libby AM, Orton HD. Prehospital and in-hospital mortality: a comparison of intentional and unintentional traumatic brain injuries in Colorado children. Arch Pediatr Adolesc Med 2005;159:665-70 CrossRef Medline

6. Jenny C, Hymel KP, Ritzen A, et al. Analysis of missed cases of abusive head trauma. JAMA 1999;281:621-26 CrossRef Medline

7. Vázquez E, Delgado I, Sánchez-Montañez A, et al. Imaging abusive head trauma: why use both computed tomography and magnetic resonance imaging? Pediatr Radiol 2014;44 (suppl 4):S589-603 CrossRef Medline

8. Jaspan T, Griffiths PD, McConachie NS, et al. Neuroimaging for non-accidental head injury in childhood: a proposed protocol. Clin Radiol 2003;58:44-53 CrossRef Medline

9. Ginde AA, Foianini A, Renner DM, et al. Availability and quality of computed tomography and magnetic resonance imaging equipment in U.S. emergency departments. Acad Emerg Med 2008;15: 780-83 Medline

10. Hedlund GL, Frasier LD. Neuroimaging of abusive head trauma. Forensic Sci Med Pathol 2009;5:280-90 CrossRef Medline

11. Kemp AM, Jaspan T, Griffiths J, et al. Neuroimaging: what neuroradiological features distinguish abusive from non-abusive head trauma? A systematic review. Arch Dis Child 2011;96:1103-12 CrossRef Medline

12. Kelly AB, Zimmerman RD, Snow RB, et al. Head trauma: comparison of MR and CT-experience in 100 patients. AJNR Am J Neuroradiol 1988;9:699-708 Medline

13. Zimmerman RA, Bilaniuk LT, Farina L. Non-accidental brain trauma in infants: diffusion imaging, contributions to understanding the injury process. J Neuroradiol 2007;34:109-14 CrossRef Medline 
14. Adamsbaum C, Rambaud C. Abusive head trauma: don't overlook bridging vein thrombosis. Pediatr Radiol 2012;42:1298-1300 CrossRef Medline

15. Hedlund GL. Subdural hemorrhage in abusive head trauma: imaging challenges and controversies. J Am Osteopath Coll Radiol 2012;1:23-30

16. Bradford R, Choudhary AK, Dias MS. Serial neuroimaging in infants with abusive head trauma: timing abusive injuries. J Neurosurg Pediatrics 2013;12:110-19 CrossRef Medline

17. Tanoue K, Aida N, Matsui K. Apparent diffusion coefficient values predict outcomes of abusive head trauma. Acta Paediatr 2013;102: 805-08 CrossRef Medline

18. Colbert CA, Holshouser BA, Aaen GS, et al. Value of cerebral microhemorrhages detected with susceptibility-weighted MR imaging for prediction of long-term outcome in children with nonaccidental trauma. Radiology 2010;256:898-905 CrossRef Medline

19. Sieswerda-Hoogendoorn T, Boos S, Spivack B, et al. Abusive head trauma, part II: radiological aspects. Eur J Pediatr 2012;171:617-23 CrossRef Medline

20. Babikian T, Tong KA, Galloway NR, et al. Diffusion-weighted imaging predicts cognition in pediatric brain injury. Pediatr Neurol 2009; 41:406-12 CrossRef Medline

21. Galloway NR, Tong KA, Ashwal S, et al. Diffusion-weighted imaging improves outcome prediction in pediatric traumatic brain injury. J Neurotrauma 2008;25:1153-62 CrossRef Medline

22. Schaefer PW, Huisman TA, Sorensen AG, et al. Diffusion-weighted MR imaging in closed head injury: high correlation with initial Glasgow coma scale score and score on modified Rankin scale at discharge. Radiology 2004;233:58-66 CrossRef Medline

23. Rappaport BA, Suresh S, Hertz S, et al. Anesthetic neurotoxicity: clinical implications of animal models. $N$ Engl J Med 2015;372: 796-97 CrossRef Medline

24. Yu CK, Yuen VM, Wong GT, et al. The effects of anaesthesia on the developing brain: a summary of the clinical evidence. Version 2. F1000Res 2013;2:166 CrossRef Medline

25. Patel DM, Tubbs RS, Pate G, et al. Fast-sequence MRI studies for surveillance imaging in pediatric hydrocephalus. J Neurosurg Pediatr 2014;13:440 - 47 CrossRef Medline

26. Iskandar BJ, Sansone JM, Medow J, et al. The use of quick brain magnetic resonance imaging in the evaluation of shunt-treated hydrocephalus. J Neurosurg 2004;101:147-51 Medline

27. Ashley WW Jr, McKinstry RC, Leonard JR, et al. Use of rapid-sequence magnetic resonance imaging for evaluation of hydrocepha- lus in children. $J$ Neurosurg 2005;103(2 suppl):124-30 CrossRef Medline

28. Forbes KP, Pipe JG, Karis JP, et al. Brain imaging in the unsedated pediatric patient: comparison of periodically rotated overlapping parallel lines with enhanced reconstruction and single-shot fast spin-echo sequences. AJNR Am J Neuroradiol 2003;24:794-98 Medline

29. Miller JH, Walkiewicz T, Towbin RB, et al. Improved delineation of ventricular shunt catheters using fast steady-state gradient recalled-echo sequences in a rapid brain MR imaging protocol in nonsedated pediatric patients. AJNR Am J Neuroradiol 2010;31: 430-35 CrossRef Medline

30. Missios S, Quebada PB, Forero JA, et al. Quick-brain magnetic resonance imaging for nonhydrocephalus indications. J Neurosurg Pediatr 2008;2:438-44 CrossRef Medline

31. Penzkofer AK, Pfluger T, Pochmann $Y$, et al. MR imaging of the brain in pediatric patients: diagnostic value of HASTE sequences. AJR Am J Roentgenol 2002;179:509-14 CrossRef Medline

32. Ba-Ssalamaha A, Schick S, Heimberger K, et al. Ultrafast magnetic resonance imaging of the brain. Magn Reson Imaging 2000;18: 237-43 CrossRef Medline

33. Singh RK, Smith JT, Wilkinson ID, et al. Ultrafast MR imaging in pediatric neuroradiology. Acta Radiol 2003;44:550-57 CrossRef Medline

34. Griffiths PD, Wilkinson ID, Patel MC, et al. Acute neuromedical and neurosurgical admissions: standard and ultrafast MR imaging of the brain compared with cranial CT. Acta Radiol 2000;41:401-09 CrossRef Medline

35. Rozovsky K, Ventureyra EC, Miller E. Fast-brain MRI in children is quick, without sedation, and radiation-free, but beware of limitations. J Clin Neurosci 2013;20:400-05 CrossRef Medline

36. Mehta $\mathrm{H}$, Acharya J, Mohan AL, et al. Minimizing radiation exposure in evaluation of pediatric head trauma: use of rapid MR imaging. AJNR Am J Neuroradiol 2016;37:11-18 CrossRef Medline

37. Fleiss JL. Measuring nominal scale agreement among many raters. Psychol Bull 1971;76:378-82 CrossRef

38. Tong KA, Ashwal S, Holshouser BA, et al. Hemorrhagic shearing lesions in children and adolescents with posttraumatic diffuse axonal injury: improved detection and initial results. Radiology 2003; 227:332-39 CrossRef Medline

39. Goske MJ, Applegate KE, Boylan J, et al. The Image Gently campaign: working together to change practice. AJR Am J Roentgenol 2008;190: 273-74 CrossRef Medline 